\title{
University infrastructure as vector of region sustainable development
}

\author{
E.Pokrovskaya \\ Department of Foreign Languages \\ Tomsk State University of Control Systems and \\ Radioelectronics \\ Tomsk, Russia \\ pemod@yandex.ru
}

\author{
M.Raitina \\ Department of Philosophy and Sociology \\ Tomsk State University of Control Systems and \\ Radioelectronics \\ Tomsk, Russia \\ raitina@mail.ru
}

\begin{abstract}
The article considers conceptual bases of the formation of innovative adaptation interaction model: "society outer social group". An outer social group is articulated as migrant students of various nationalities from various ethnic groups. Under this model, the leading role is assigned to the Centre, incorporated into the University infrastructure. The authors adhere to the international approach in understanding the problems of cross-cultural interaction and emphasize the development of personal potential and creative abilities. The model suggested in the article is the core for sustainable development of the region. The complexity of the model was confirmed by the method of structural-genetic synthesis and the methodology of systems analysis.
\end{abstract}

Keywords-university model; outer social group; effective intercultural interaction

\section{INTRODUCTION}

In the last decade, the model of universities, focused on creating innovative infrastructure in order to solve the task of embedding in the economic sector and functioning as a modern educational research, cultural, educational, information and communication center is actively formed in Russia. This University model, aimed at creating a positive for self-realization cultural and educational environment of the University, was developed in Tomsk State University of Control Systems and Radioelectronics (TUSUR). In this context, a key task of the University today is its growing influence on the development of economy and social sphere of the city, region and wider Asian part of Russia.

One of the strategic breakthrough in University infrastructure is the first all-Siberian cross-cultural center (hereinafter - Center) formed on the basis of the Humanitarian faculty to provide a competitive inclusion of the University in the global educational trends. The idea of the Center was not random. For many years, at the Humanitarian faculty and its departments (Department of philosophy and sociology and Department of foreign languages), researches in the field of intercultural dialogue and interethnic interaction were carried out. Currently leading scientists of the faculty deal with the synthesis of technical and humanitarian knowledge. It is important for the protection of the individual from external information and communication impact, maintaining emotional stability and harmonic communicative context. The increase of the sources and means of information, the diversity of their interpretations, including those depending on mental, ethnic models of the world, "blur" individual space of life goals of the subjects of communication. Therefore, in educational discourse, it is necessary to create an innovative methodology for the keeping comfortable communicative context and protection of the individual from the harmful effects of communication. It demands basic approaches conceptualization that provides information differentiation, assign only the necessary part for a person, correlate with moral, spiritual attitudes of the individual. All this was the basis for the further development of humanitarian ideas of the scientific school and it was extrapolated to the creation of the Center.

\section{THEORY AND METHODS}

The Center, incorporated in the infrastructure cluster of the University, is a socio-economic, educational phenomenon. It concentrates both full-time and remote resources on several key areas of the human being associated primarily with the problems of survival of the individual in the discrete world, the humanization of society, humanization of cultural values, socio-psychological environment of the University. On the grounds of the Centre there is a selection of the best social startups on the topic of self-realization in the sociohumanitarian sphere and extrapolation of project ideas in the world scientific and educational space. The intention of globalization is characterized by the desire for separation of purposes of human activity from the present, actually existing reality in the direction of unification leads to the loss of the meaning of social existence. On the one hand, one can easily imagine the widespread dissemination of scientific, medical, military and other achievements, but not always able to represent the dynamics of human relationships and changes in the balance between goals and means of human activity. On the other hand, the concept of regionalization is reflected in the preservation of identity of national cultures and semantic codes. Realizing this, let us go to the next level of interaction, level of axiological evaluations of socio-cultural processes in the modern world, and in this context personal fulfillment may act as the measuring instrument, which is required for the transition to this level. 
A personal potential (hereinafter - PP) is presented as an integral characteristic of a level of personal maturity. Different psychological approaches have identified phenomenology, reflecting the effects of the PP or its failure with the help of such notions as will, power, Ego, internal support, locus of control, orientation on action, and some others. However, all the mentioned concepts, though are most directly related to PP, describe only some of its faces. When one speaks on a personal potential, it is not so much about the underlying personality traits or attitudes, but about the peculiarities of the system organization of personality in general, its architectonics that is based on a complex scheme of mediation. The authors believe that in the structure of personality it is impossible to allocate a separate factor of PP, but it is possible to isolate the vector of individual potency. Based on the results of the study, let us highlight the ontological issue of emergence of the system when it is quite difficult to identify individual factors in the integral characteristics, but there are directions that lead to the actualization of a factor in the composition of the entire system.

With the aim of optimizing the resources of the individual, let us use the project method, allowing implementing a system interaction of the structural components. In the late 19th century and early 20th century, scientific thinking led to a pragmatic turn and actualized project-based learning. Project management began to emerge in the 50-s in the USA, and a decade later it was widely used in the West. As well as to the West, in Russia, the project management was in the 60-s, but the development was quite different.

In the West and in other developed countries, the project management has received a wide range of development at once; it was used in many fields, such as entrepreneurship. In the USSR, there was a completely different socio-economic system, and market of the project management tools was not up to it. Project management could develop in Russia only in theory, because there was no implementation in practice. This situation could only be explained by the predominance of state ownership in the USSR and prevailing administrativecommand methods of management organizations and the state in general.

Currently, project management is used in Russia quite actively, but still has a negative side. On the other hand, there are the pros of project management:

- it allows one to keep the economy on the level of the developed countries;

- it allows one to co-operate with the CIS countries and Eastern Europe;

- it allows one to use the experience of the West;

- it allows one to develop national project management, which would be easier to implement in Russia.

The main stages of development of project management include:

- fundamentals of project management;

- network planning methods and management;
- the use of computers for the management of specific projects;

- organization management (multi-project control);

- integrated management system;

- modern methods of professional management on the basis of transfer and adaptation of the Western experience.

The project method is a basic one for the Center functioning. It is effectively used in the sphere of educational projects and dual network platforms' creation that provides scientific novelty of the research undertaken in the context of the activities of the Centre as the object of the University's infrastructure.

1. For the first time in the domestic and global scientific practice, the authors set the task of the solution of complex social and humanitarian issues related to the establishment of information and communication cluster in the humanitarian science school to protect individuals from information and communication extremism in the external environment. Under the cluster, the authors mean the integrity of hardware and software, psycho-pedagogical and marketing systems. Currently developed teaching methods and the outlined history of educational technology of protecting the individual from the harmful communication impact allow the communication entities to "start" the trigger - the trigger for their own emotional safety; it is theoretically the essence of the information-communication extremism.

2. One of the trending educational projects of the Center is to create a network of dual sites. Resources, developed elearning content for international dual platforms in the field of protection of the individual in terms of identifying hidden threats and potential internal resources of the individual in a situation of the discreteness of the world have no analogues in Russia and abroad.

The novelty of the dual network project is provided with synergistic content, pedagogical tools used in combination with the unique diagnostics, correction and filtration of aggressive information flow.

3. An international website that concentrates information about scientific schools, including research and educational resources, interactive communication platform for webinars, video labs and etc. is being created. Scientific novelty and significance of the research lies in the ideology of replenishment of the content of the portal through the placement of materials research, performed at the request of the strategic partners-organizations of the University and of dual sites. Genres of research vary from analytics, research papers to video/audio field studies and focus groups.

4. To promote humanitarian scientific schools on the basis of the first all-Siberian cross-cultural center, a media holding company is established. It is designed to promote the ideas of humanism, unity, positive interethnic interaction, and operating in the territory of Asian part of Russia. The function of the holding company includes the production and broadcast of the information-analytical programs on the topic of research. Members of the staff of the scientific school are public figures, producers of educational and cultural programs 
on the theme of ethno-cultural education, inter-ethnic harmony, combating xenophobia.

Thus, the development of high-tech complex of Russian cross-cultural center aimed at concentration of resources, their incorporation into the regional economy will serve as an educational foresight, ensure the transfer of technologies developed at the dual sites at the global level.

\section{RESULTS AND DISCUSSION}

The one of the unique results of the Center functioning is the interaction model "universities - national-cultural autonomies (NCA) - government" in the field of ethnocultural education.

Key mission of the Center is the formation of a single ethno-educational environment, the establishment of dialogue between representatives of different nationalities or religions, universities, schools and local authorities. N.P. Bezuglova noted that "formation of intercultural competence helps to open new horizons for joint activity of people of different cultural backgrounds" [1]. In this case, an important strategic line, which the cross-cultural Center heads for, is to create an interaction between universities, NCA and government through the complex innovative creative practices in the problem-oriented and project-organised education [2].

The scheme presented in Fig. 1 simulates the formation of adaptive educational space on the basis of complex interactions. The mechanism of interaction of key elements of the adaptation model is presented in Fig. 2. This mechanism implies overlap zones of the semantic fields of society (the interaction model "universities - national-cultural autonomies (NCA) - government") and outer social groups through cocreativity of all participants of educational space.

Let us note that the new socio-economic development strategy of the city "Tomsk 3.0" focused on the creation of an image of Tomsk as a comfortable place to stay creative and competitive.

The pragmatic turn in culture affects cross-cultural processes and actualizes those conceptual models which assess the methodological bases and principles of education, focus mechanisms and ways of learning creativity, creation [3].

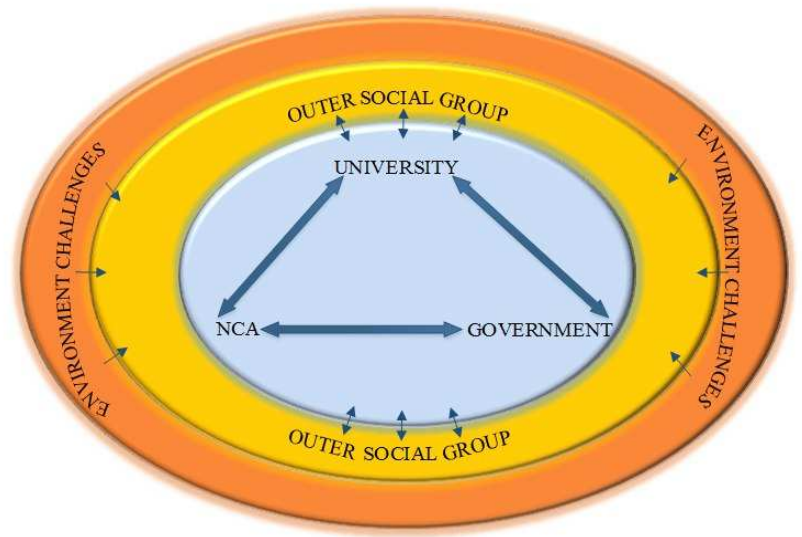

Fig.1. Innovative adaptation model of interaction "society - outer group"
B.S. Gershunskyi, forming a model of the philosophy of education for XXI century, notes that "the creation process is always creative" [4], and the purpose of education is to develop a real ability to create. The latter imposes certain requirements for pedagogical theory that needs to be changed under the influence of modern socio-cultural context.

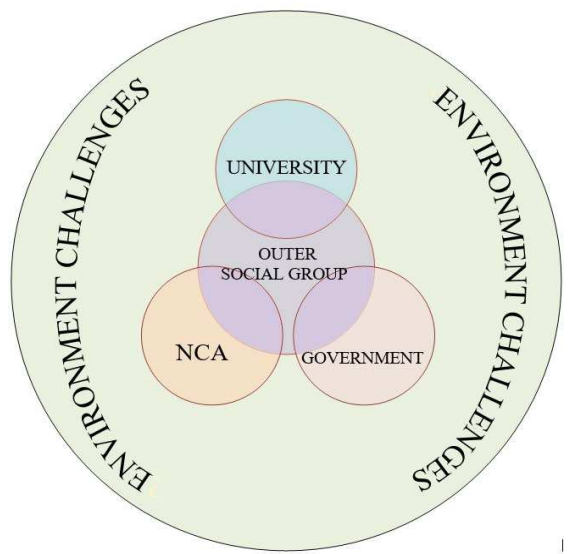

Fig. 2. The mechanism of interaction of key elements of the adaptation model

With time, these changes are updated basing on the concept of the variety of pedagogical practices. Certain rules and principles of teaching work are formulated, the immanent pedagogy of creativity is formed through a variety of pedagogical practices similarly to the situation in the sphere of artistic creativity. The latter causes certain intentions, justified in the framework of pedagogical theory, learning theories creativity [5]. Such synthesis of theoretical and immanent pedagogy of creativity may be a smart toolkit, the so-called the pedagogy poetics of creativity. An example of learning poetics can be "didactic multidimensional technology", developed by V.E. Steinberg [6], which presents the rules and activates the basic mechanisms of thinking within the visual and conceptual models of the educational process. Thus, the pragmatic turn in the conceptualization of creativity generates the intention on the development of the pedagogy of creativity, the formation of the pedagogy poetics of creativity as the synthesis of philosophy and practical pedagogy creativity in the formation of the operational structure of creative thinking.

In this regard, the determining factor in the formation of the brand is the development of socio-educational space by bringing in a city of students, including those from neighboring countries, Caucasus and Asia. In addition, in the framework of the project "InoTomsk2020" student campus is being constructed; the infrastructure of business incubators is functioning. Among them, TUSUR was the first one. This project involves most talented young people in Tomsk universities. This is especially relevant in view of promotion Tomsk as a unique scientific-educational center, where in a small area there are six state universities, and it is shaping the educational and intellectual elite of the country [7]. However, according to opinion polls it was revealed that studentsmigrants of different ethnicity (outer social group) arriving in Tomsk, often experience difficulty in learning, in communicating with the student community and teachers not 
only during training sessions but also in extra-curricular life. Foreign students form ethnic communities, as a consequence, are poorly integrated into the socio-cultural environment of the University and the town, which inevitably leads to failure, they will feel marginalized. In this regard, it is becoming very urgent to solve the problem of interaction of high schools with NCA, which stand for newly arrived foreign students as a kind of conductor for the society. Thus, the study [8] established that the representatives of the Tajik nationality as the migrants have relatively "good" image and a portrait, mental health, help the economy of the region and contribute to ethnic harmony at the expense of such of their dispositions as goals in life, autosympathy, propensity for mutually useful and enjoyable contacts with other people, which is a necessary basis for the synergistic orientations. However, the picture is not so perfect: according to the factor analysis, it is evident that there is orientation "to itself", and this indicates the presence of internal conflict between the values of their own culture, belonging to a collectivist type of cultures, and circumstances of the surrounding world, which hinders the development of PP. As a result, one need the response to the challenges of modern time, namely the need for mechanisms how to remove these contradictions. One of the most popular solutions is the above-described Center and the program of the person's self-realization, developed by the authors, which is based on the cultural and value needs. Let us note the causes of the increasing interaction between NCA and universities.

1. In accordance with modern sociocultural situation in Russia, it is changing educational interest, requests of the local population and potential students - representatives of different nationalities. This factor had a direct impact on the educational space of the city and, as a consequence, the development of cooperation between educational institutions and national-cultural autonomies. Each of them is interested in attracting to teaching all students, including those from other regions and countries.

2. On the other hand, national-cultural autonomies are conducting special vocational work among the population of neighboring countries for universities potential applicants in Tomsk.

3. Some NCA organize courses on studying Russian language and culture, which contribute to the most favorable integration of foreign students in the town society and the educational environment of universities and other educational institutions. Representatives of autonomies purposefully accompany their students throughout the learning [9].

Scientific and practical novelty in this case is justified by the fact that NCA and local authorities carry out a new function, feel their necessity in the educational process. The contemporary higher education is characterized by the transformation of ideas about the competencies that the graduates acquire. This is largely due to implementation of new generation federal educational standards based on the competence approach, which today is the trend of the study of education applied aspects in relation to the needs of the economy and society, specifying the tasks of education, where the content of education is supplemented by interdisciplinary co-holding [10]. Trends of globalization in modern educational environment determine the necessity of the interdisciplinary integration of educational resources and programs in Russian education. For example, an educational organization in conjunction with the NCA will develop and implement programs and projects in the field of ethno-cultural education. Until now, NCA have not actualized their potential participation in the educational process of schools and universities. Modernization of the educational process, new generation federal educational standards, including higher education, set tasks to develop students' cultural competencies that contribute to the development of inter-ethnic harmony. Among these competencies there are the following: respect for historical heritage and cultural traditions; a willingness to be racial, national, ethnic and religious tolerance; the ability and willingness to carry out their activities in different spheres of social life accepted in society moral norms and values. This raises the need for a new way to deploy educational content. The active inclusion in the process of developing programs of study of the representatives of the NCA makes it possible to solve pedagogical challenges and achieve educational goals through the use of full instrumentation, the designated in new generation federal educational standards, including interactive methods and forms of work within the classroom. In turn, the authorities are not outside observers but actively involved in the development of educational infrastructure. For example, the Committee on local government of Tomsk authorities supported the project "Organization of interaction of nationalcultural autonomies with the universities in the city of Tomsk for the formation of interethnic tolerance in the Siberian region", the intention of which emerged at the round table discussion "University infrastructure of Tomsk - a resource efficient cross-cultural interaction", in Cross-cultural youth forum with international participation "Ethno-cultural mosaic".

This event included eleven educational town venues with the following forms of work: plenary session; lecture; design marathon; creative labs; workshops; communication workshops for young people; a discussion club on the issues of overcoming xenophobia and harmonization of interethnic relations; tournament for the national sports games; video conference with representatives of regions of Russia on issues of interethnic consent; workshops on the grounds of ethnocultural centers of education; round tables; concert program with participation of representatives of national-cultural autonomies. Over 400 people attended the Cross-cultural forum. All forum events were covered in numerous TV reports, radio interviews, publications in social networks, presentations at conferences and scientific articles. TUSUR students organized the Festival of national cultures. Here are some examples of interactive sites at the festival. So, the students attended public lectures by leading experts in the field of intercultural communication, participated in discussions, talked about movies the national perspective on the cinema club "Live screen", competed in tournaments, national sports games "Sibmiks" etc. The participants of this educational event were able to learn to dance a traditional dance - Buryat dance "Ekhor", to play in the Yakut national game "Habilik", to try on the traditional Russian costumes of XVIII - XIX centuries, to make the ancient Altai decoration, to participate in the Tuva national wrestling "Huresh". In addition to active 
games and national dances, the students acquainted with the life and lifestyle of the German and Tatar peoples on the territory of Tomsk region. Culture-ethnic communities staged a mobile exhibition and talked to everyone about their cultures. So after communion to their national traditions was the immersion in the cultures of different ethnic groups.

Experience with educational migrants has allowed us to determine some of the most effective ways of working:

- individual counseling on various matters (administrative, housing, psychological, etc.);

- seminars and clubs (support groups);

- creative and sports associations.

These measures have proved effective, as they could contribute to overcoming existing barriers in the primary adaptation and integration of migrants to a new sociocultural environment. However, experience has shown that the number of migrants attending these events is around 40-50\% and almost completely depends on personal motivation to solve the existing problems and the desire to become part of a new social and cultural environment. In addition, activities that focus only on work with migrants, contribute to the formation of a comfortable environment within the group of migrants, which creates a vacuum that prevents communication outside of the group. Highlighted problems promoted the search for new ways of interaction that would allow solving the obstacle in working with migrants. As an example of one of these new forms we note supervision as a means of organizing effective interaction with educational migrants.

In addition, on the positive side of supervising activities, which are applicable for work with migrants are:

- organization of interaction between migrants and indigenous students in an informal environment;

- inclusion of migrants in the process of interaction, visits to various events (training, cultural, leisure, lecture, etc.) is more than $95 \%$;

- no need to create a new administrative formation.

\section{CONCLUSION}

In conclusion it is necessary to point out the following:

- the Center as a part of the University infrastructure is an instrument of effective organization and coordination of creation of harmonious interethnic relations in the internal environment of the University;

- the Center aims at the development of legislative, programmatic materials for the formation of an integral internal environment of the University, comfortable for interethnic cooperation on the territory of a region;

- the outer social group in the context of cross-cultural communication is adapted to the host community with the Centre;

- the proposed innovative adaptation interaction model: "society - outer social group" will help to build effective management of the ethno-social processes with the aim of sustainable development of region economy;

- it is necessary to carry out the monitoring and examination of existing successful interactions at specific local sites for the purpose of extrapolating them at regional, federal and international level;

- the main obstacle in the development of the University infrastructure is the lack of a methodological framework for the cluster systems' management in the context of innovative interdisciplinary projects.

Thus, the University infrastructure with comfortable psychological environment, tolerant communicative space for both migrants and the host community is becoming the driving force and the resource of effective intercultural interaction. In this sense, the first all-Siberian cross-cultural center serves as a modern educational research, cultural, educational, information and communication complex, raising the general cultural competence of students and humanizing potential of the person in whole.

\section{Acknowledgment}

The work is performed with the support of the Ministry of Education and Science of the Russian Federation, project no. 28.8279.2017/8.9

\section{References}

[1] N.P. Bezuglova, "The value basis of intercultural communication", Culture and civilization, 2013, no. 3-4, pp. 14-24.

[2] I.B. Ardashkin, N.V. Martyushev, V.P. Bezborodov, "Problem Methodology as One of the Ways of Innovative Organization of Educational Process", Procedia - Social and Behavioral Sciences, 166, Proceedings of The International Conference on Research Paradigms Transformation in Social Sciences 2014 (RPTSS-2014), pp. 227-231, 16-18 October 2014.

[3] P.K. Engelmeier, "Theory of creativity", SPb, Education, 1910.

[4] B.S. Gershunskyi, "Education as the religion of the third Millennium: the harmony of knowledge and faith", M., Pedag. Soc. in Russia, 2001.

[5] P. Papava, "From controlled freedom to freedom of governance. Development of critical thinking in higher education: technology and approaches", M., ZGL, 2007, pp. 15-21.

[6] V.E. Shteinberg, "Didactic multidimensional technology: a chronicle of the development", Pedagogical journal of Bashkortostan, 2011, no. 5, pp. 74-84.

[7] A.A. Kornienko, "Adaptation Problems Experienced by International Students in Aspect of Quality Management", The European Proceedings of Social \& Behavioural Sciences (EpSBS), 2016, no. 19, pp. 358-361. http://dx.doi.org/10.15405/epsbs.2017.01.48 http://earchive.tpu.ru/handle/11683/37270

[8] E.M. Pokrovskaya, O.V. Gorskikh, M.Y. Raitina, L.V. Smolnikova, A.V. Larionova, N.P. Lyzhina, S.O. Dongak, A.V. Kolesnik, "Selfrealization of migrants: salvation tools", Tomsk, RCRO, 2015.

[9] O.V. Gorskikh, E.M. Pokrovskaya, T.I. Suslova, L.V. Smolnikova, "University infrastructure of Tomsk - a resource efficient intercultural interaction", Tomsk, TUSUR, 2013.

[10] Yu.Sh. Sirazitdinova, A.A. Dulson, B. Mueller, "Project management practices in engineering university", IOP Conference Series: Materials Science and Engineering, 2015, no. 93. http://dx.doi.org/10.1088/1757899X/93/1/012080 http://earchive.tpu.ru/handle/11683/20091 